\title{
Design Child-friendly Food Guide Based on Nutritional Risk Assessment of Pediatric Inpatients
}

\author{
Rabab El-Sayed Hassan El-Sayed* \\ Pediatric Nursing Department, Faculty of Nursing, Mansoura University, Egypt \\ *Corresponding author: biboelsayed@gmail.com
}

Received December 06, 2018; Revised January 22, 2019; Accepted February 18, 2019

\begin{abstract}
Background: However, the hospital malnutrition's prevalence is high; this condition is ignored and, as a result, is not treated. The first step in fighting malnutrition is to know how to identify patients at higher risk of suffering from this condition by using risk screening tools. The aim of the present study was to design child-friendly food guide based on nutritional risk assessment of pediatric inpatients. Method: In this exploratory cross-sectional study, one hundred children in medical departments affiliated to Mansoura University Children Hospital, Egypt was assessed as regarding their admission and discharge weight and height of the body, the body mass index/ $\mathrm{kg} / \mathrm{m}^{2}$, and biochemical analysis of hemoglobin and serum albumin. A Screening Tool for Assessing Malnutrition in Pediatrics (STAMP) has been applied in order to also assess pediatric inpatients' overall risk of malnutrition. Furthermore, the perspective of hospitalized children/their mothers about the hospital introduced food habits and meals pattern were surveyed. Results: Slightly less than half of the studied children were males and the large percent of the hospitalized children's mothers were highly educated. According to STAMP score, all studied children were considered to be at risk for malnutrition with the underlying diseases were related to neurological or general medical disorders among the largest. The study findings also revealed that the majority of participants tried to escape the delivered hospital meals and reported their needs for a dietary guide contains healthy food varieties to overcome the risk of undernutrition related to children's hospitalization. Conclusion: every effort must be made to identify children at risk of developing under-nutrition upon admission in order to provide necessary nutritional support for them.
\end{abstract}

Keywords: child-friendly food guide, malnutrition risk, pediatric inpatients, STAMP

Cite This Article: Rabab El-Sayed Hassan El-Sayed, "Design Child-friendly Food Guide Based on Nutritional Risk Assessment of Pediatric Inpatients.” American Journal of Nursing Research, vol. 7, no. 2 (2019): 208-218. doi: 10.12691/ajnr-7-2-13.

\section{Introduction}

The provision of quality nutritional care is a key element of good health care. All pediatric patients are entitled to safe and nutritious food. Their nutritional needs are expected to be met during hospitalization. The positive effects of providing nutritional support have been documented in different clinical situations. While some patients may benefit from special nutritional support techniques (enteral or parenteral), most are based on ordinary hospital food to improve or maintain their nutritional status in order to optimize their recovery from the disease. The American Society for Parenteral and Enteral Nutrition has defined pediatric malnutrition as "an imbalance between nutrient requirements and intake, resulting in a cumulative deficit of energy, protein or micronutrients that can negatively affect growth, development and other relevant results” [1]

Hospitalized children can easily lose weight, unless special attention is paid to this problem. The disease usually leads to an increase in consumption of the energy, while interfering with the appetite of children and intake of energy. Malnutrition during hospitalization increases the vulnerability of sick children to acquiring a nosocomial infection, which interferes with their recovery. The late recovery is combined to increase both the time a patient spends in the hospital and the stay's cost. Furthermore, hospital malnutrition in children can lead even more to growth retardation and negative educational and social outcomes; as well as contributing to premature death. [2]

Children and young people with special health care needs must undergo routine screening for malnutrition in admission facilities. Registered dietitian nutritionists (RDNs) or nurses in pediatric units and all of the healthcare team members must work together to ensure that the detection of malnutrition becomes an integral part of routine pediatric care. Although studies are limited, malnutrition in this population may lead to more complicated hospitalizations due to progression of the underlying disease or condition, poor wound healing, or return to the level of previous activity, complications that can significantly increase the length of stay and cost of hospitalization. [3,4] Comprehensive assessment and nutritional intervention for pediatric patients may reduce the need for more expensive hospitalization by addressing 
nutritional deficits that may predispose the patient to an acute illness or aggravate the underlying disease or condition. [5]

In the developing countries, acute malnutrition's prevalence in hospitalized children remains remarkably high, but there is a lack of data on nutritional status during hospitalization. During their stay, the focus is mainly on the primary medical problem. Little attention is still given to the nutritional status of the child. The management and prevention of malnutrition in hospitalized children is based on the early identification of those at risk in order to implement early nutritional interventions. To this end, various risk assessment tools have been developed to identify children at risk of developing malnutrition. [6,7,8,9] The prevalence of under-nutrition in hospitalized children has varied according to socioeconomic status and medical environment, ranging from $6.1 \%$ to $37 \%$. [10] However, studies related to this issue are still lacking in Egypt [11].

The European Society of Nutrition and Clinical Metabolism, the American Society of Parenteral and Enteral Nutrition and the European Society of Pediatric Gastroenterology, Hepatology and Nutrition recommend a quick and easy nutritional examination; to determine in advance patients who are at nutritional risk. It was reported that several validated pediatric nutritional risk screening tools were effective in identifying children at risk of malnutrition. [12] The Pediatric Malnutrition Assessment Screening Tool (STAMP) is a validated nutritional screening tool for use in children who are hospitalized and their age from 2 to 16 years old. [13]

Under-nourished hospitalized children do not respond to medical treatment in the same way as if they were wellnourished. In addition, they are much more likely to die, with or without health problems, than their well-nourished counterparts. For that, pediatric inpatients need specialized attention and have diversified demands for adequate growth and development, and thus need a different approach and interest in nutritional assessment and supply. Therefore, the current study outcome was to design a guide booklet that illustrates healthy dietary choices and proper composition of various meals, and distribute it for hospital authorities, dietary management and residents. This approach can help in the proper growth and recovery of hospitalized children with nutritional imbalances or nutritional needs [14] through set out simple, specific instructions, taking into consideration the differences among medical diagnosis and caloric needs, to overcome the problem of under-nutrition due to hospital admission and meet the caregivers' needs of providing adequate food of high quality for their children during their illness.

\subsection{Problem Statement}

Under-nutrition in hospitalized children is a very important pathological condition and a risk factor for unfavorable outcomes, prolonged hospital stay, late recovery and increased costs of treatment. The reduction of dietary intake, together with the increase of energy requirements are the main causes of hospital under-nutrition. The reported prevalence of acute under-nutrition in admitted infants and children from different countries ranges from 6.1 to $40.9 \%$. In children with an underlying disease, higher prevalence of chronic malnutrition
(44-64\%) was reported. [15] Although most studies have documented the prevalence of under-nutrition at hospital admission time, only a few studies have assessed the development of under-nutrition among children during their hospital stays. These studies reveal that the nutritional status deteriorates in $5 \%$ to $27 \%$ of children after hospital admission. [12]. Therefore, early risk detection of developing under-nutrition in pediatric hospitalized patients may prevent or reduce nutritionassociated complications and prolonged hospitalization.

\subsection{Aim}

This study was aimed to design child-friendly food guide based on nutritional risk assessment of pediatric inpatients.

Specific objectives

1- Assess the nutritional status of hospitalized children to detect those at risk for developing under-nutrition.

2- Assess hospitalized children/their caregivers' (mothers) opinions about hospital food habits and meals pattern introduced through the period of hospital stay.

\subsection{Research Questions}

1- Are pediatric inpatients at risk for developing undernutrition throughout their hospital admission period?

2- What are children/their mothers' perspectives about hospital dietary habits and the delivered meals pattern throughout the period of hospital stay?

\section{Patients and Method}

\subsection{Patients}

\subsubsection{Research Design}

An exploratory cross-sectional design was used in this study.

\subsubsection{Setting}

The study was conducted at all medical departments affiliated to Mansoura University Children Hospital (MUCH), Egypt. The hospital building is composed of 7 floors that received mixed diagnosis of pediatric inpatients. This institution is the only specialized tertiary care referral and teaching hospital in Mansoura City that contained 135 beds.

\subsubsection{Study Sample}

A convenience sample of pediatric inpatients between 2-18 years old and their caregivers (mothers), who were admitted to the study setting and accept to participate in the study from July to December 2017 ( $n=100)$.

\section{Power analysis}

A power analysis identified an appropriate sample size of minimum 96 participants by using a sample size calculation (confidence level of 90\%, 0.12 SD effect size).

\section{Exclusion criteria}

- Immobilized patients who could not be weighed (e.g., severe burns, fractures, or abdominal trauma) 
- Patients admitted for less than 7days

- Patients who had conditions which affected hydration

- Patients who were clinically unstable or critically ill and on parenteral nutrition

- Patients who their caregivers could not read and write

\subsubsection{Tools of Data Collection}

Data were collected by using the following 3 tools: 2.1.4.1. Tool (I): A Structured Interview Questionnaire Sheet

It was developed by the researcher after reviewing the related current literature. It was concerned with the participants' demographic characteristics, the quality of food provided in the hospital relatively to home, hospital menu analysis, as well as hospital's food habits from the perspective of hospitalized children/their mothers as the following:

2.1.4.1.1. Part 1: It included questions about the characteristics of participants' children and their caregivers, such as gender, age, birth order, level of education, admission and discharge body weight and height, body mass index (BMI) $\mathrm{kg} / \mathrm{m}^{2}$, weight gain or decline during hospital stay, date of admission and discharge, and residence as well as the mothers' level of education, and their occupations.

2.1.4.1.2. Part 2: It covered the child's medical history, including diagnosis per hospital admission section, duration of current hospital stay, and near past history of hospitalization; including if admitted during the year before, as well as frequency, and duration of hospitalization(s) in the last year.

2.1.4.1.3. Part 3: This part was concerned with providing detailed information on current and recent changes in dietary intake compared with the child's eating and dietary habits as reported by older child or the child's caregiver. It included questions about the hospital food pattern introduced in the 3 main meals, number of the provided daily meals including snacks, as well as description from the caregiver to a sample of foods and drinks that a hospitalized child took daily.

2.1.4.1.4. Part 4: It was consisted of questions related to hospital introduced food habits from the perspective of hospitalized children/their mothers that inquired about if the hospital provided meals are healthy, if the hospital provided well balanced meals, adequacy of food amount, the quality of food introduced, if hospital meals contained the essential food groups, and if the caregiver tried to escape hospital food (why and how). Finally the participants' children and their caregivers were asked about their needs to overcome the problem of under-nutrition due to hospitalization.

\subsubsection{Tool (II): The Nutritional Status Assessment Tool}

The Screening Tool for Assessment of Malnutrition in Pediatrics (STAMP) was adopted and applied to assess pediatric inpatients' overall risk of malnutrition. Originally, it was developed by a team from Royal Manchester Children's Hospitals and the University of Ulster, the lead investigator being Helen McCarthy, Lecturer and Honorary Pediatric Dietitian. [16] STAMP is made up of 5 main steps, which are: screening form that is composed of 5-steps, diagnosis table, weighing and measuring instructions, and centile quick reference tables. The full nutritional assessment classified pediatric inpatients as 'at high risk' or 'medium risk' or 'low risk' of under-nutrition if obtained a score of $\geq 4$ or 2-3 or $0-1$ respectively in STAMP. Risk of under-nutrition was considered if a child presented with one or more of a low weight percentile relative to height percentile and age; sub-optimal dietary intake over the recent past that would be unlikely to improve in the next 3-5 days; and a clinical history/treatment plan that might result in either increased metabolic stress, decreased dietary intake or increased nutritional losses indicated by continuous weight decline.

\subsubsection{Tool III: Patient's Nutritional Biomarkers Profile}

It was involved obtaining and recording the results of routinely drawn blood samples from hospitalized children for biochemical analysis of hemoglobin and serum albumin at admission and on discharge.

\subsection{Method}

\subsubsection{Preparation Phase}

- An official letter was issued from the Faculty of Nursing to the Director of Mansoura University Hospital to permit for the researcher conducting the current study.

- Tools were formulated after thorough review of literature. The study developed tools and the designed guide were reviewed for their content validity by five experts in the field of pediatric clinical nutrition and necessary modifications were done accordingly.

- The STAMP had substantial reliability (inter-rater reliability: $\kappa: 0.752$; intra-rater reliability: $\kappa: 0.635)$. When compared with dietetic assessment as a reference standard, STAMP had a sensitivity of $83.3 \%$, specificity of $66.7 \%$ and an overall agreement of $76.5 \%$ [17]

- At data collection time, the researcher was introduced herself and a simple explanation about the aim of the study was given to the participants' children or their mothers.

\section{- Ethical consideration}

An approval was obtained from the Research Ethics Committee of Faculty of Nursing, Mansoura University. The participants were informed that all questionnaires will be anonymous and the collected data will be treated confidentially. A written consent was obtained from the participant child's mother after providing detailed information about the nature of the study and its consequences before collection of the required data. Older children and their mothers were assured that their participation is voluntary and they have the right to withdraw at any time from the study, and their perspectives about the provided hospital food would have no effect on hospital services they received.

\section{- The Study Guide Construction Process}

A child-friendly food guide for pediatric inpatients and/or their caregivers plus the hospital authorities including the nutrition consultant, was designed by the researcher based on extensive review of related current literature and pilot study obtained results to reduce the risk of under-nutrition among hospitalized children. It was revealed that, nutritional issues for pediatric inpatients 
include poor quality of cooked food; dull method food presentation, in addition to poor intake of fruits, vegetables and micronutrients such as calcium. Therefore, the booklet content addressed model and provides a healthy diet guide for pediatric inpatients. The guide illustrates that it is important that pediatric meals are nutritious, appetizing and tempting to sick children. The guide also clarifying that, child food should have a balance between healthy options and familiar or favorite foods, as well as a range of appropriate choices should be available on the pediatric menu.

\subsubsection{Exploratory Phase}

\section{Pilot study}

It was conducted on 10 hospitalized children of different medical diagnoses (10\% of the study sample). They were excluded from the study sample, as they test the tool applicability. Based on the finding of the pilot study, the study dietary guide was designed.

\section{Operational phase/fieldwork}

This was a multi-days cross-sectional foodservice exploratory survey that was conducted over three months from February to April 2018 at the University Children Hospital in Mansoura City. All children who met the inclusion criteria and hospitalized for a period of 7 or more days were included in the study. Demographic and medical data, together with a questionnaire for the current and previous dietary habits, were collected during a structured interview with patients and (when required) with their caregivers. Pediatric patients/caregivers were assessed with the use of a set of questions that considered assessing resident satisfaction and the nutritional adequacy, and under-nutrition risk. Measurements of anthropometric measures (weight and height) were performed within the first $24 \mathrm{~h}$ after admission, repeated on weekly base through the period of hospital stay, and measured at discharge by the researcher who used the STAMP weighing and measuring instructions. While the body mass index was calculated once, the laboratory tests of hemoglobin and serum albumin were performed twice to compare between the results of on admission and at discharge.

After STAMP step 1 question that determined whether the child's condition has any nutritional implications, and step 2 question that concerned with the child's current nutritional intake were answered, the growth charts or the centile quick reference tables for girls and boys were used by the researcher to determine the child's measurements ( $>3$ or $>2$ or 0 to 1 centile spaces) to be used to assign a score for step 3 of STAMP. Then, the total score of STAMP screening form and classification of malnutrition risk (low, medium, or high) was determined for each study participant (STAMP step 4) and integrated in his/her questionnaire sheet.

The survey was comprised of open and closed-ended questions in an attempt to provide a comprehensive picture of the current state of menu evaluation in Mansoura University Children Hospital, while recognizing the food preparation and distribution systems, menus, and resources among the hospital. The order of the questions allowed the participants to answer straightforward and less contentious questions first to decrease the propensity for defensiveness. The final open ended question allowed the pediatric inpatient/caregiver to describe the breakfast, lunch, and dinner meals and snacks that were introduced daily in the hospital menu. For each patient, the survey and measurements were completed in 40 to 60 minutes. The steps of each tool were completed by the same investigator (researcher) in the same order. STAMP screening form step-5 was omitted; in which any decisions on providing nutritional treatment were not made because the hospital staff/policy did not include dieticians.

\subsection{Data Collection Technique}

At the beginning of the interview, the researcher explained the questionnaire three main parts, the anticipated length of the interview and that the participants' responses would be written down. As the interview proceeded, the researcher used a friendly conversational tone, asked one question at a time, attempted to remain neutral, encouraged responses by remaining silent or using a consistent non-leading non-bias probes, and transitioned between the three parts. The researcher probed when the participants were not forth coming, repeated the question when requested and when the participant did not directly answer the question. At the end of the interview the researcher thanked the participant and inquired whether he/she would like a copy of the booklet.

The collected data were recorded in the questionnaire sheet then captured on an Excel spreadsheet along with quantitative data. Excel version 14.4 .8 (2011) was used to prepare the collected data for analysis.

\subsection{Statistical Analysis}

The collected data were revised, coded, tabulated, and analyzed using the statistical package for the social sciences (SPSS, version 23; IBM SPSS Statistics, Chicago, IL, USA). Descriptive statistics were generated for the included sample population using counts and proportions to characterize the study findings, and Paired sample t test was used to compare between the obtained data. An independent sample t-test and one-way ANOVA were used to study mean difference between generated scores for general impression about hospital's food habits, and hospital's meals pattern. P value threshold of 0.05 was deemed as significant.

\section{Results}

The characteristics of the patients (Table 1) showed that $54 \%$ of them were females, and $40 \%$ aged from 5 to less than 10 years old. The mean age of the participants' children was $6.97 \pm 4.18$ years with the range of $2-17$ years. In these patients, the mean height was $112.9 \pm 24.4 \mathrm{~cm}$ with the range of $65-164 \mathrm{~cm}$, and the mean body mass index (BMI) score on admission time was $18.4 \pm 4.5 \mathrm{~kg} / \mathrm{m}^{2}, 38 \%$ were firstborn son or daughter, and $51 \%$ attended primary and elementary schools. This table also elucidated that $45 \%$ of the patients' mothers were highly educated, 52\% of them were housewives, and $54 \%$ of the participants were urban residents. 
Regarding to current and near past history of hospitalization, Table 2 illustrated that the most frequent cause responsible for pediatric admission in this study was related to neurological disorders (23\%), and the mean hospital stay of patients was $20.44 \pm 10.47$ days with a range of 8 to 62 days. Moreover, most (97\%) of the study sample were admitted to the hospital the year before data collection; $50.5 \%$ of them were hospitalized for 3 times or more and $60.8 \%$ stayed in the hospital between 30 to less than or equal 60 days.

According to STAMP score, Figure 1 clarified that $82 \%$ of pediatric inpatients were classified as high risk, and $18 \%$ as moderate risk for malnutrition.

A total of 100 hospitalized children who are considered to be at risk for malnutrition according to STAMP score were grouped based on their underlying disease as shown in Table 3, in which $24.4 \%$ and $27.8 \%$ of who were either high risk for malnutrition or medium risk for malnutrition suffered from neurological disorders or general medical disorders respectively.

Table 4 explained pediatric inpatients or their mothers' perspectives about hospital food habits, in which 53\%, and 54\%, positively reported that the hospital introduced healthy and well balanced meals respectively. On the other hand, $100 \%$, and $70 \%$, stated that the hospital did not introduce any snacks, and the provided 3 meals were inadequate and of bad quality compared to homemade food respectively. Therefore, the majority (86\%) of the participants tried to escape hospital meals, because its preparation was very bad from the perspective of $70.9 \%$ of the studied sample, so that $64 \%$ tried to deliver homemade food, and 68\% declared that they need dietary guide contains healthy food varieties to overcome under-nutrition caused by hospitalization.

In relation to pediatric inpatients or their mothers' perspectives about hospital's meal pattern, Table 5 showed that 30\% positively announced that the breakfast and dinner contained all the essential food elements for healthy growth and development. On the opposite side, $20 \%$ and $47 \%$ of the participants reported that the hospital provided breakfast and lunch mainly composed of carbohydrates, and had little protein, vitamins, and calcium, as well as $25 \%$ and $30 \%$ complained that no food varieties were found in the lunch and dinner meals served by the hospital.

Table 6 showed the difference between admission and discharge nutritional biomarkers among the participants' children. There were significant differences among pediatric inpatients as regarding their weight, and laboratory investigations of hemoglobin and serum albumin at $\mathrm{P}<.001$.

Table 7 revealed positive associations between overall nutritional biomarkers of participants' children on time of their hospital discharge and different aspects attached to hospital provided food and meal pattern, regardless the duration of hospital stay. These results suggest that hospitalized children's discharge body weight, hemoglobin, and serum albumin reduction linked with the duration of previous hospitalization in the year before, and providing well balanced, healthy meals and of high quality food during hospital stay at $\mathrm{P}=.017, .002,0.17$, and .003 respectively.

Table 1. Characteristics of Hospitalized Children and their Mothers (n=100)

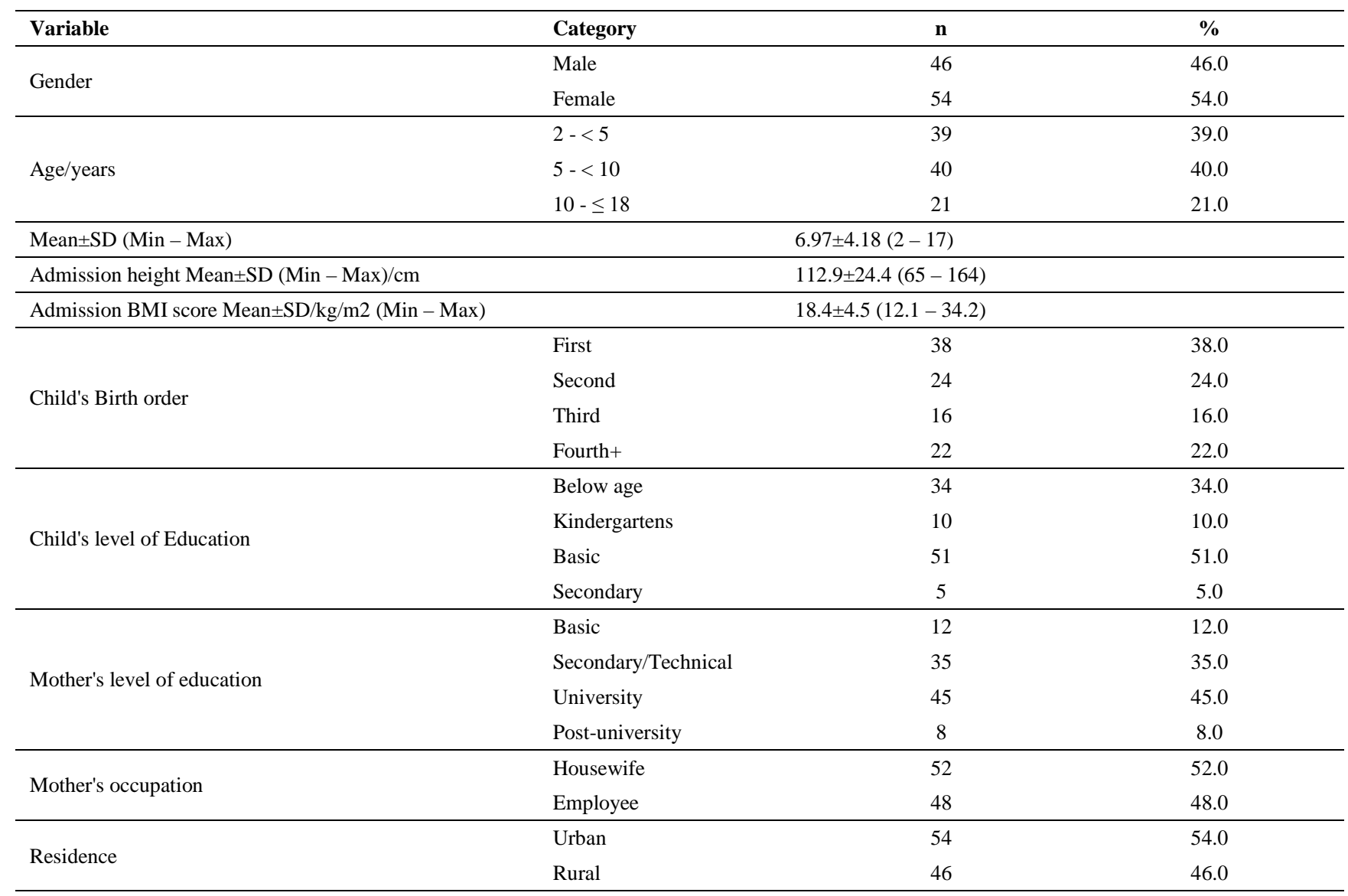


Table 2. Distribution of Pediatric Inpatients according to their Current and Near Past History of Hospitalization

\begin{tabular}{|c|c|c|c|}
\hline Variable & Category & $\mathrm{N}$ & $\%$ \\
\hline \multirow{8}{*}{ Current Hospital admission/department } & - Neurological disorders & 23 & 23.0 \\
\hline & - Cardio/Chest infection & 21 & 21.0 \\
\hline & - Gastroenteritis & 15 & 15.0 \\
\hline & - Endocrinology & 14 & 14.0 \\
\hline & - General medicine disorders & 12 & 12.0 \\
\hline & - Tropical & 7 & 7.0 \\
\hline & - Hematology & 4 & 4.0 \\
\hline & -- Nephrology & 4 & 4.0 \\
\hline \multirow{3}{*}{ Current duration of hospital stay/days } & $>7-30$ & 92 & 92.0 \\
\hline & $31-60$ & 4 & 4.0 \\
\hline & $>61$ & 4 & 4.0 \\
\hline Mean \pm SD (Min - Max) & $20.44 \pm 10.47$ & & \\
\hline \multirow{2}{*}{ Admitted to the hospital during the year before $(\mathrm{n}=100)$} & Yes & 97 & 97.0 \\
\hline & No & 3 & 3.0 \\
\hline \multirow{4}{*}{ Number of hospital admission(s) during the year before $(\mathrm{n}=97$ ) } & Once & 6 & 6.2 \\
\hline & Twice & 27 & 27.8 \\
\hline & Three times & 15 & 15.5 \\
\hline & More than 3 times & 49 & 50.5 \\
\hline \multirow{3}{*}{ Total admission days spent in the year before (n=97) } & $<30$ & 25 & 25.8 \\
\hline & $30-\leq 60$ & 59 & 60.8 \\
\hline & $>60$ & 13 & 13.4 \\
\hline
\end{tabular}

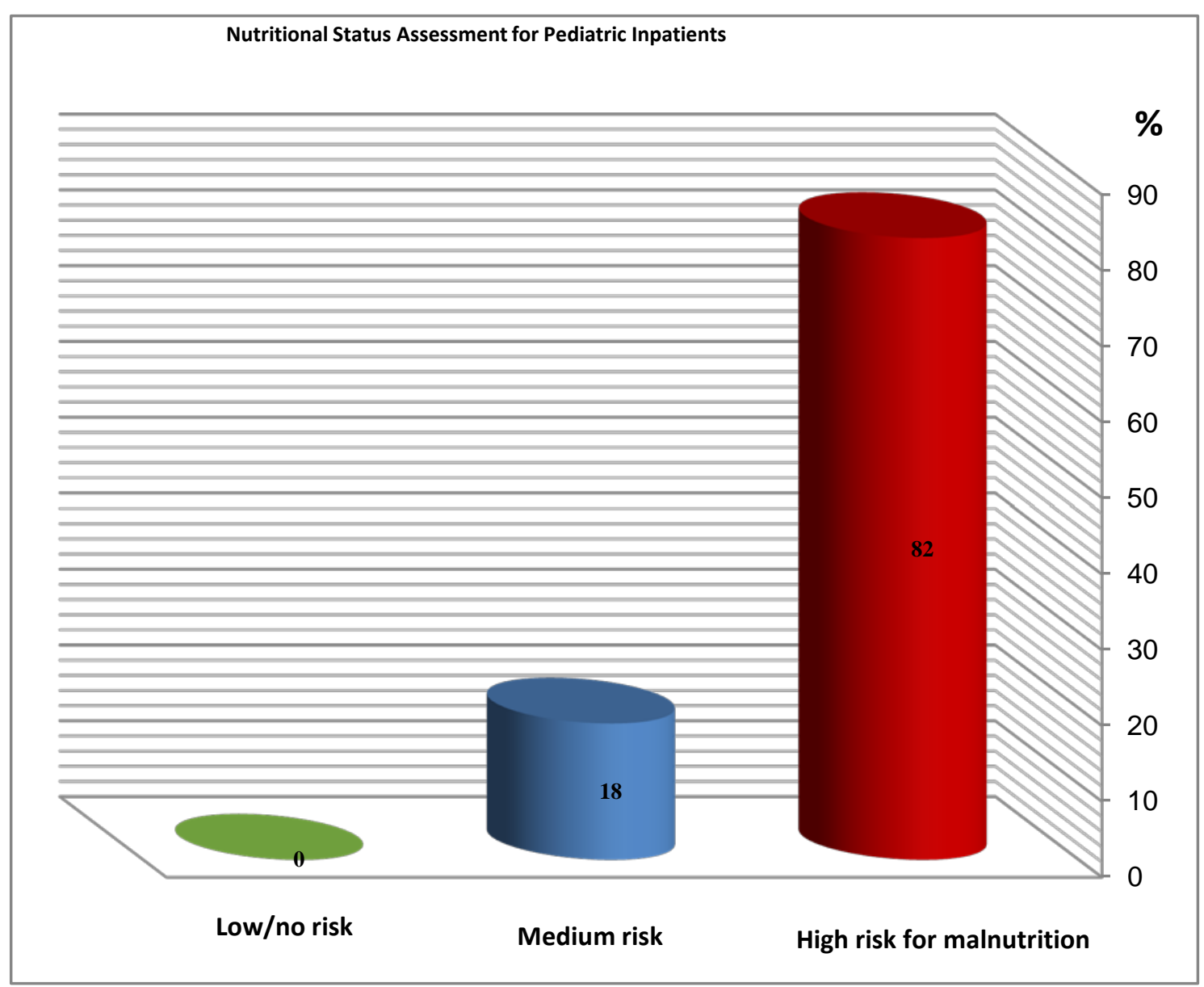

Figure 1. Classification of Hospitalized Children according to STAMP Score 
Table 3. The Prevalence of Malnutrition Risk among the Participants' Children based on the Underlying Disease ( $\mathrm{n}=\mathbf{1 0 0})$

\begin{tabular}{lcccc}
\hline \multirow{2}{*}{ Underlying disease } & \multicolumn{2}{c}{ High risk for malnutrition (n=82) } & \multicolumn{2}{c}{ Medium risk for malnutrition (n=18) } \\
\cline { 2 - 5 } & $\mathbf{N}$ & $\mathbf{\%}$ & $\mathbf{N}$ & $\mathbf{\%}$ \\
\hline Neurological disorders & 20 & 24.4 & 4 & 16.6 \\
Cardio/Chest infection & 17 & 20.7 & 2 & 22.2 \\
Gastroenteritis & 13 & 15.9 & 0 & 11.1 \\
Endocrinology & 14 & 17.1 & 5 & 0.0 \\
General medicine disorders & 7 & 8.5 & 1 & 27.8 \\
Tropical & 6 & 7.3 & 2 & 5.6 \\
Hematology & 2 & 2.4 & 1 & 11.1 \\
Nephrology & 3 & 3.7 & 5.6 \\
\hline
\end{tabular}

Table 4. Distribution of Pediatric Inpatients/their Mothers according to their Perspective about Hospital's Food Habits

\begin{tabular}{|c|c|c|c|}
\hline Variable & Category & $\mathrm{N}$ & $\%$ \\
\hline \multirow{2}{*}{ The hospital introduced healthy meals } & - Yes & 47 & 47.0 \\
\hline & - No & 53 & 53.0 \\
\hline \multirow{2}{*}{ The hospital introduced balanced diet } & - Yes & 46 & 46.0 \\
\hline & - No & 54 & 54.0 \\
\hline \multirow{2}{*}{ Provided daily snacks } & - No & 100 & 100.0 \\
\hline & - Yes & 0 & 0.0 \\
\hline \multirow{2}{*}{ Adequacy of food items in the three meals } & - Inadequate & 70 & 70.0 \\
\hline & - Adequate & 30 & 30.0 \\
\hline \multirow{3}{*}{$\begin{array}{l}\text { The quality of food provided in the hospital } \\
\text { relatively to homemade }\end{array}$} & - Getting worse & 70 & 70.0 \\
\hline & - The same quality & 22 & 22.0 \\
\hline & - Getting better & 8 & 8.0 \\
\hline \multirow{2}{*}{ Try to escape hospital meals } & - Yes & 86 & 86.0 \\
\hline & - No & 14 & 14.0 \\
\hline \multirow{3}{*}{ Why $(n=86)$} & - Contains harmful food elements & 10 & 11.6 \\
\hline & - Very bad in its preparation & 61 & 70.9 \\
\hline & - Tasteless & 15 & 11.4 \\
\hline \multirow{2}{*}{ How $(n=86)$} & - Deliver homemade food & 55 & 64.0 \\
\hline & - Buy external food & 31 & 36.0 \\
\hline \multirow{2}{*}{$\begin{array}{l}\text { Children/caregivers needs to overcome under- } \\
\text { nutrition due to hospitalization }\end{array}$} & - Provide dietary information through professionals & 32 & 32.0 \\
\hline & - Provide a dietary guide contains healthy food varieties & 68 & 68.0 \\
\hline
\end{tabular}

Table 5. Distribution of Pediatric Inpatients/their Mothers according to their Perspectives about Hospital's Meal Pattern (n=100)

\begin{tabular}{|c|c|c|c|c|c|c|}
\hline \multirow{2}{*}{ Variable } & \multicolumn{2}{|c|}{ Breakfast } & \multicolumn{2}{|c|}{ Lunch } & \multicolumn{2}{|c|}{ Dinner } \\
\hline & $\mathbf{N}$ & $\%$ & $\mathbf{N}$ & $\%$ & $\mathbf{N}$ & $\%$ \\
\hline Contain all the essential food elements & 30 & 30.0 & 25 & 25.0 & 30 & 30.0 \\
\hline Mainly carbohydrates, \& little protein, vitamins \& calcium & 20 & 20.0 & 47 & 47.0 & 5 & 5.0 \\
\hline Mainly carbohydrates, \& little protein, vitamins & 12 & 12.0 & 3 & 3.0 & 29 & 29.0 \\
\hline Mainly carbohydrates, \& little protein \& calcium & 10 & 10.0 & 7 & 7.0 & 8 & 8.0 \\
\hline Mainly carbohydrates, \& little vitamins & 10 & 10.0 & 7 & 7.0 & 12 & 12.0 \\
\hline No varieties & 6 & 6.0 & 25 & 25.0 & 30 & 30.0 \\
\hline Imbalanced & 2 & 2.0 & 4 & 4.0 & 5 & 5.0 \\
\hline Little carbohydrates, protein, vitamins \& calcium & 4 & 4.0 & 1 & 1.0 & 1 & 1.0 \\
\hline Mainly carbohydrates, \& little protein & 2 & 2.0 & 1 & 1.0 & 6 & 6.0 \\
\hline Mainly carbohydrates, \& little vitamins \& calcium & 4 & 4.0 & 5 & 5.0 & 4 & 4.0 \\
\hline
\end{tabular}

Table 6. The Difference between Admission and Discharge Nutritional Biomarkers of Hospitalized Children (n=100)

\begin{tabular}{|c|c|c|c|c|}
\hline \multirow{2}{*}{ Item } & \multirow{2}{*}{ Mean } & \multirow{2}{*}{ Std. Deviation } & \multicolumn{2}{|c|}{ Test of Sig } \\
\hline & & & $t$ & $p$ \\
\hline Admission weight $/ \mathrm{kg}$ & 25.07 & 14.346 & \multirow{2}{*}{10.698} & \multirow{2}{*}{$<.001 *$} \\
\hline Discharge weight/kg & 23.52 & 14.343 & & \\
\hline Admission hemoglobin (gm/dl) & 9.55 & 1.160 & \multirow{2}{*}{5.993} & \multirow{2}{*}{$<.001 *$} \\
\hline Discharge hemoglobin (gm/dl) & 9.18 & 1.035 & & \\
\hline Serum albumin on admission (g/dl) & 4.18 & .933 & \multirow{2}{*}{8.295} & \multirow{2}{*}{$<.001 *$} \\
\hline Serum albumin on discharge (g/dl) & 3.69 & .728 & & \\
\hline
\end{tabular}

* Statistically significant at $\mathrm{p} \leq 0.05$. 
Table 7. Relation between Discharge Nutritional Biomarkers and Dependent Variables of Hospitalization and Provided Food Habits $(\mathbf{n}=100)$

\begin{tabular}{lcc}
\hline Variables & \multicolumn{2}{c}{ Significance } \\
\hline \multirow{2}{*}{ Total hospital admission days spent in the year before } & $r$ & 4.261 \\
& $P$ & $\mathbf{. 0 1 7}$ \\
Current duration of hospital stay & $r$ & .805 \\
& $P$ & .40 \\
The hospital introduced well balanced meals & $r$ & 3.203 \\
The hospital introduced healthy meals & $P$ & $\mathbf{. 0 0 2 *}$ \\
The hospital food served is of high quality & $r$ & 4.225 \\
& $P$ & $\mathbf{. 0 1 7}$ \\
\end{tabular}

r: Pearson coefficient $*$ Statistically significant at $\mathrm{p} \leq 0.05$.

\section{Discussion}

Pediatric hospitals usually provide food to hospitalized patients, despite the nutritional content of the food provided has not been evaluated. Nutritional status is essential for the management of hospitalized children and is directly related to the length of hospitalization. The goal of the hospital's food supply system is to offer regular and nutritionally adequate therapeutic menus, while balancing the budget and operational capacity. However, the introduction of poor quality menu items with inadequate or unhealthy nutritional content, resulting inpatient poor intake, and subsequently increases the risk of undernutrition among hospitalized children. The results of this study revealed that more than half of the studied children were girls, two fifths aged from 5 to less than 10 years old, and more than two fifths of mothers of hospitalized children were highly educated. These findings were similar to those of a recent study entitled "The prevalence of under-nutrition upon hospitalization in children in a developing country: A single hospital study from Malaysia" except in gender, as more than half (58\%) were found to be males. [18]

Furthermore, our findings were consistent with those of a recent study about "Assessing the Prevalence and Treatment of Malnutrition in Hospitalized Children in Mofid Children's Hospital During 2015-2016", [19] which demonstrated that the mean height was $114.7 \pm$ $21.9 \mathrm{~cm}$ with the range of $69-175 \mathrm{~cm}$ and the mean BMI score at time of admission was $16.4 \pm 4.4 \mathrm{~kg} / \mathrm{m}^{2}$. From the researcher's point of view these similarities could be attributed to similarity of the participants' age group (2-18 years), so they had look like anthropometric measurements.

The results of the present study support previous findings that the underlying disease or the cause for hospitalization is related to loss of weight during the stay, which is often increased by inflammation. In this study, about one quarter of patients who were admitted because they suffered from neurological disorders; including meningitis and encephalitis were at high risk for malnutrition, and more than another one quarter of patients who were admitted because they suffered from general medical disorders were at medium risk for malnutrition. According to Lheureux and Preiser 2017, [20] the body inflammatory response during acute phase of the disease is associated with high initial expenditure of energy and excretion of nitrogen. Mehta and colleagues 2013, [1] added that the disease often induces anorexia and fever, in addition to other factors that are related to hospital stay, such as procedures that require fasting, hospital diet acceptance, and both quality and quantity of the served hospital diet.

Concerning the duration pediatric patients stayed in the hospital, the study findings reported that the vast majority of children were admitted for more than one week to one month. There are some studies investigating the association between nutritional risk scores and the length of hospital stay, in developed [17,21,22] and developing $[23,24,25]$ countries. Others have found that a hospital stay for more than five days is considered a risk factor for malnutrition. [26,27,28] The length of hospital stay is multifactorial. The introduction of ordinary meals is often postponed, and fewer children receive food on the first day of their hospitalization. Another problem is length of hospital stay is negatively related to patient satisfaction with hospital meals. [29,30,31] According to the researcher experience, the hospital food system is introducing the same meals for almost all patients, with the exception of diabetics, regardless the calories and nutritional needs of the patients. Moreover, from the researcher's point of view, the failure to identify patients at risk of malnutrition on admission and, consequently, appropriate nutritional therapies that are rarely prescribed for malnourished patients is another factor of malnutrition. Thus, nutritional risk assessment for hospitalized children must include many factors in addition to the anthropometric parameters. Healthcare teams and the hospital administrators should also pay attention to special nutritional needs of patients.

In the present study, STAMP clarifies patients with the highest probability of under-nutrition during hospitalization, revealed that more than four fifths of patients were classified as high risk for malnutrition (Figure 1). Although studies [32,33,34] have shown that some nutritional assessment tools especially STAMP, are accurate in detecting nutritional risk among hospitalized children from developed countries, and the authors concluded that the tools work well, there is no evidence that nutritional screening actually improves their nutrition related outcome. In their study [35], suggested that future research should focus on demonstrating a better outcome of a nutritional screening program in hospitals that include both screening and an associated nutritional intervention. In my opinion, design child-friendly food guide for hospitalized children and its distribution on their caregivers is a very relevant solution that could ensure a varieties of appropriate, case tailored, healthy, and balanced meals be brought during the hospital admission period until complete recovery at home.

According to the results, less than three quarters of the participants mentioned that the amount of hospital food delivered in the 3 main meals (breakfast, lunch, and dinner) was not enough. This finding disagrees with the result of [30] who disclosed that $75.7 \%$ of patients mentioned that the hospital introduced enough food amounts. In addition more than two thirds of hospitalized children or their mothers were reported that the hospital provided food of bad quality, which was similar with another study finding 
that indicated that patients were scored food quality as the lowest, because there was less variety in the menu [36], which is also compatible with the current study findings (Table 5). This can be explained by the fact that in most Egyptian public hospitals, no menus were distributed; hence, patients do not have choices on what will be served. Another explanation is the repetition or no variety of menus practiced in hospitals. Moreover, menu-related issues have been identified as influential factors on patients' food consumption, and therefore, they should be improved otherwise, patients will try to escape hospital meals, which occurred among more than four fifths of the current study participants. Apart from that, taste, flavors, and how the meat and vegetables were cooked also affected patient satisfaction with the dimension of food quality, which were also explained by more than two thirds of pediatric inpatients and their mothers as "the hospital meals are very bad in its preparation" and more than one fifth of them as "tasteless".

The study results were able to find significant differences at $\mathrm{P}<.001$ between hospitalized children's nutritional discharge biomarkers and that upon admission (Table 6). Thus, the present study confirms that under-nutrition is a common problem in children who require hospital admission care, which may be related to food habits followed by the hospital. Our findings are in concordance with [37] who found that there were positive correlations between anthropometric measurements; including body weight, biochemical indicators (hemoglobin and serum albumin), dietary intake and malnutrition score among hemodialysis patients. In the same context, another two studies conducted by $[38,39]$ concluded that impaired appetite due to food preparation issues, inadequate food intake, recent weight loss and reduction in anthropometric measurements were identified as major contributors of malnutrition. These two studies also evaluates the association between malnutrition risk and anthropometric data, nutrition related parameters, and length of hospital stay, reported positive relations; which is go in the same line with the current study findings except it did not find a correlation between hospitalized children's nutritional biomarkers and length of hospital stay (Table 7). Actually, "no appetite" is a common statement given by pediatric patients when they do not eat, and the researcher has viewed that patient's food consumption can be improved by enhancing the quality of provided food, which by indirect way positively affects the anthropometric measurements and biochemical readings regardless hospitalization period.

Feedback from surveys concerned with patients' perceptions about services provided within healthcare facilities is necessary for hospital administrators to determine operational strengths and weaknesses for continuous quality improvement. In addition, the analysis of data derived from the patient's opinion is a valuable tool, not only to increase profitability, but also to be used as a guide for strategy development by researchers, so that the child-friendly food guide was designed on this basis. Nowadays, patient requests have increased as they seek better services than ever before. It is crucial for academic staff hand by hand with hospital administrations to improve the quality of health service to specifically meet what patients want.

\section{Limitations of the study}

The study's sampling process included voluntary patient/mother participation in completing the survey about the introduced hospital meals, which can contribute to nonparticipation bias. A second limitation of the study was the possibility that patients/mothers might not join the study participants during their hospital stay because of fear of affecting the level or quality of care they receive while in the hospital. A statement was included as a heading on the questionnaire assuring participation in the survey was voluntary and their responses would not affect care and would remain confidential.

\section{Conclusion}

Nutritional imbalance that happens during hospital stay, is very frequent but little studied. Its genesis is related to many factors; some are inherent to the pediatric patient, and others, to the lodging. Every effort must be made to early identify young children who are at risk of developing under-nutrition upon hospitalization and providing immediate nutritional support. Managing hospital-acquired malnutrition requires the healthcare team and hospital authorities to consider nutrition as part of hospital care. There are two strategies to combat hospital under- nutrition by the pediatric nurses, which are nutritional risk screening and providing child-friendly food guide.

\section{Approaches for Improvement}

The hospital nutrition consultant and hospital director were contacted and a meeting was conducted, so that the researcher presented the study results and the designed guide booklet. Moreover, and based on the study findings, the researcher recommended through a discussion with the hospital authorized persons some ideas aimed to improve the hospital food habits and prevent under-nutrition among pediatric inpatients. The elaborated ideas were concentrated around the importance of malnutrition screening, which should be included as part of patients' standard care, provide various nutritious meals on the menu for the hospitalized children, the caloric intake should be calculated in the distributed meals so that it suits the child's stage of growth and development and the child's medical diagnosis, snacks should be provided for pediatric inpatients, and enrich the hospital provided meals with minerals, vitamins and micronutrients.

\section{Recommendations}

The researcher also recommended some items to be considered by hospital director in developing universal nutritional assessment for pediatric inpatients as the following:

- Create a nutrition team: Ideally this should include nurses, dieticians, pharmacists and pediatricians.

- Develop a hospital policy on regular measurement and plotting of child's height and weight by trained staff and validated equipment.

- Adopting one of the published tools as a method of nutrition screening for hospitalized children. 
- Ensure protocols are in place for the management of hospitalized children at nutrition risk.

- Establish a weekly nutrition ward round: A dedicated time each week for evaluation of how children at risk are progressing with nutritional management will help increase the priority of nutrition for all patients.

Audit: Important to have baseline audit results and demonstrate improvement in quality and patient outcomes.

Further study may be required to assess the effect of applying child-friendly food guide menus on reducing the risk of developing malnutrition among critically ill pediatric patients.

\section{Acknowledgements}

The cooperation of the hospital manager and the participation of hospitalized children and their mothers in the study are well recognized.

\section{Conflicts of Interest Disclosure}

The author declares no conflict of interest.

\section{References}

[1] Mehta, N. M., Corkins, M. R., Lyman, B., Malone, A., Goday, P. S., Carney, L., ... \& American Society for Parenteral and Enteral Nutrition (ASPEN) Board of Directors. Defining pediatric malnutrition: a paradigm shift toward etiology - related definitions. Journal of Parenteral and Enteral Nutrition , 37(4), 460-481.2013.

[2] Hwang, E. H., Park, J. H., Chun, P., \& Lee, Y. J. Prevalence and Risk Factors for the Weight Loss during Hospitalization in Children: A Single Korean Children's Hospital Experience Pediatric gastroenterology, hepatology \& nutrition, 19(4), 269275. 2016.

[3] Hecht, C., Weber, M., Grote, V., Daskalou, E., Dell'Era, L., Flynn, D. \& Joosten, K. Disease associated malnutrition correlates with length of hospital stay in children. Clinical nutrition, 34(1), 53-59. 2015.

[4] Radman, M., Mack, R., Barnoya, J., Castañeda, A., Rosales, M., Azakie, A., ... \& Fineman, J. The effect of preoperative nutritional status on postoperative outcomes in children undergoing surgery for congenital heart defects in San Francisco (UCSF) and Guatemala City (UNICAR). The Journal of thoracic and cardiovascular surgery, 147(1), 442-450. 2014.

[5] Geier, L. M., Bekx, M. T., \& Connor, E. L. Factors contributing to initial weight loss among adolescents with polycystic ovary syndrome. Journal of pediatric and adolescent gynecology , 25(6), 367-370. 2012

[6] Mărginean, O., Pitea, A. M., Voidăzan, S., \& Mărginean, C. Prevalence and assessment of malnutrition risk among hospitalized children in Romania . Journal of health , population, and nutrition, 32(1), 97. 2014.

[7] Joosten, K. F., \& Hulst, J. M. Prevalence of malnutrition in pediatric hospital patients . Current opinion in pediatrics , 20(5), 590-596. 2008.

[8] Beser, O. F., Cokugras, F. C., Erkan, T., Kutlu, T., Yagci, R. V., Ertem, D., ... \& Coşkun, M. E. Evaluation of malnutrition development risk in hospitalized children. Nutrition, 48, 40-47. 2018.

[9] Teixeira, A. F., \& Viana, K. D. A. L. Nutritional screening in hospitalized pediatric patients : a systematic review . Jornal de pediatria, 92(4), 343-352. 2016

[10] Chourdakis, M., Hecht, C., Gerasimidis, K., Joosten, K. F., Karagiozoglou-Lampoudi, T., Koetse, H. A., ... \& Koletzko, B.
Malnutrition risk in hospitalized children: use of 3 screening tools in a large European population -3 . The American journal of clinical nutrition, 103(5), 1301-1310. 2016.

[11] SHAABAN, Sanaa. 1Faculty of Medicine, Ain Shams University, Cairo, Egypt.(Correspondence to : May Nassar : maie_nassar@ yahoo. co. uk). 2018.

[12] Beser, O. F., Cokugras, F. C., Erkan, T., Kutlu, T., Yagci, R. V., Ertem, D., ... \& Coşkun, M. E. Evaluation of malnutrition development risk in hospitalized children . Nutrition, 48, 40-47. 2018.

[13] McCarthy, H., Dixon, M., Crabtree, I., Eaton - Evans, M. J., \& McNulty, H. The development and evaluation of the Screening Tool for the Assessment of Malnutrition in Paediatrics (STAMPC) for use by healthcare staff. Journal of Human Nutrition and Dietetics, 25(4), 311-318. 2012.

[14] Yi, D. Y. Enteral Nutrition in Pediatric Patients . Pediatric gastroenterology, hepatology \& nutrition, 21(1), 12-19. 2018.

[15] Daskalou, E., Galli-Tsinopoulou, A., Karagiozoglou-Lampoudi, T., \& Augoustides-Savvopoulou, P. Malnutrition in hospitalized pediatric patients : assessment, prevalence, and association to adverse outcomes. Journal of the American College of Nutrition , 35(4), 372-380. 2016.

[16] McCarthy, H., McNulty, H., Dixon, M., \& Eaton - Evans, M. J. Screening for nutrition risk in children : the validation of a new tool. Journal of Human Nutrition and Dietetics ，21(4), 395-396. 2008.

[17] Wong, S., Graham, A., Hirani, S. P., Grimble, G., \& Forbes, A Validation of the Screening Tool for the Assessment of Malnutrition in Paediatrics (STAMP) in patients with spinal cord injuries (SCIs). Spinal cord, 51(5), 424. 2013.

[18] Lee, W. S., \& Ahmad, Z. The prevalence of under-nutrition upon hospitalization in children in a developing country: A single hospital study from Malaysia . Pediatrics \& Neonatology, 58(5), 415-420. 2017.

[19] Imanzadeh, F., Olang, B., Khatami, K., Hosseini, A., Dara, N., Rohani, P., ... \& Farahbakhsh, N. Assessing the prevalence and treatment of malnutrition in hospitalized children in Mofid Children's Hospital during 2015-2016. Archives of Iranian medicine, 21(7), 302-309. 2018.

[20] Aurangzeb, B., Whitten, K. E., Harrison, B., Mitchell, M., Kepreotes, H., Sidler, M., ... \& Day, A. S. Prevalence of malnutrition and risk of under-nutrition in hospitalized children . Clinical nutrition, 31(1), 35-40. 2012.

[21] Aurangzeb, B., Whitten, K. E., Harrison, B., Mitchell, M., Kepreotes, H., Sidler, M., ... \& Day, A. S. Prevalence of malnutrition and risk of under-nutrition in hospitalized children. Clinical nutrition, 31(1), 35-40. 2012.

[22] Huysentruyt, K., Alliet, P., Muyshont, L., Rossignol, R., Devreker, T., Bontems, P., ... \& De Schepper, J. The STRONGkids nutritional screening tool in hospitalized children: a validation study. Nutrition, 29(11-12), 1356-1361.) 2013.

[23] Peng, L. T., Li, R., Zhao, W. H., Chen, Y. H., Li, X. M., Chen, M. Y., ... \& Li, X. N. Nutritional risk screening and its clinical significance in 706 children hospitalized in the surgical department. Zhongguo dang dai er ke za zhi= Chinese journal of contemporary pediatrics, 15(10), 880-885. 2013.

[24] Li, X. Reply of Letter to the Editor-Nutritional risk screening and its clinical significance in hospitalized children. Clinical Nutrition, 33(2), 372. 2014.

[25] Moeeni, V., Walls, T., \& Day, A. S. Assessment of nutritional status and nutritional risk in hospitalized Iranian children . Acta Paediatrica, 101(10), e446-e451. 2012.]

[26] Campanozzi, A., Russo, M., Catucci, A., Rutigliano, I., Canestrino, G., Giardino, I., ... \& Pettoello-Mantovani, M. Hospital-acquired malnutrition in children with mild clinical conditions . Nutrition, 25(5), 540-547.| 2009.

[27] Quadros, D. R. S., Kamenwa, R., Akech, S., \& Macharia, W. M. Hospital-acquired malnutrition in children at a tertiary care hospital. South African Journal of Clinical Nutrition, 31(1), 8-13. 2018.

[28] Weimann, A., Braga, M., Carli, F., Higashiguchi, T., Hübner, M., Klek, S., ... \& Waitzberg, D. L. ESPEN guideline : clinical nutrition in surgery. Clinical nutrition, 36(3), 623-650. 2017.

[29] Theurer, V. A. Improving patient satisfaction in a hospital foodservice system using low -cost interventions : Determining whether a room service system is the next step.| 2011. 
[30] El Sherbiny N. A., Ibrahim E. H., \& Hewedi M. M. Patients’ satisfaction with delivered food services in Fayoum Hospitals . EC Nutr, 9(2), 94-104. 2017.

[31] Abdelhafez, A. M., Al Qurashi, L., Al Ziyadi, R., Kuwair, A., Shobki, M., \& Mograbi, H. Analysis of factors affecting the satisfaction levels of patients toward food services at General Hospitals in Makkah ， Saudi Arabia . American Journal of Medicine and Medical Sciences, 2(6), 123-130; 2012.

[32] Huysentruyt, K., Devreker, T., Dejonckheere, J., De Schepper, J., Vandenplas, Y., \& Cools, F. Accuracy of nutritional screening tools in assessing the risk of undernutrition in hospitalized children. Journal of pediatric gastroenterology and nutrition 61(2), 159-166. 2015.

[33] Teixeira, A. F., \& Viana, K. D. A. L. Nutritional screening in hospitalized pediatric patients : a systematic review . Jornal de pediatria, 92(4), 343-352., 2016.

[34] Chourdakis, M., Hecht, C., Gerasimidis, K., Joosten, K. F., Karagiozoglou-Lampoudi, T., Koetse, H. A., ... \& Koletzko, B. Malnutrition risk in hospitalized children: use of 3 screening tools in a large European population -3. The American journal of clinical nutrition, 103(5), 1301-1310. 2016.
[35] Huysentruyt, K., Devreker, T., Dejonckheere, J., De Schepper, J., Vandenplas, Y., \& Cools, F. Accuracy of nutritional screening tools in assessing the risk of undernutrition in hospitalized children. Journal of pediatric gastroenterology and nutrition 61(2), 159-166. 2015.

[36] Aminuddin, N. F., Vijayakumaran, R. K., \& Abdul Razak, S. Patient Satisfaction With Hospital Foodservice and its Impact on Plate Waste in Public Hospitals in East Malaysia . Hospital Practices and Research, 3(3), 90-97. 2018.

[37] Ling, L. H. L., \& Mun, C. Y. Correlations between anthropometric measurements, biochemical indicators, dietary intake and Dialysis Malnutrition Score among haemodialysis patients in Sibu Sarawak. Malaysian Journal of Nutrition, 24(2)! 2018.

[38] Yalçın, T., Yürük, A. A., Türkoğlu, İ., Ilgaz, F., Açıkgöz, A., Aksan, A., ... \& Samur, G. Nutritional status and food intake are related to malnutrition risk and length of stay in hospitalized patients. Progress in Nutrition, 20(3), 438-446. 2018.

[39] Tsaousi, G., Panidis, S., Stavrou, G., Tsouskas, J., Panagiotou, D., \& Kotzampassi, K. Prognostic indices of poor nutritional status and their impact on prolonged hospital stay in a Greek university hospital. BioMed research international, 2014. 